Supporting Information

\title{
Effect of Nanogap Morphology on Plasmon Coupling
}

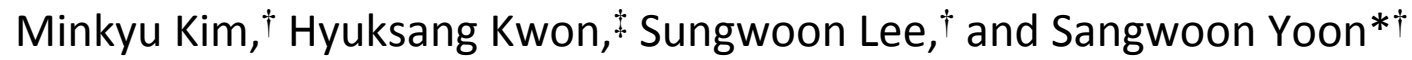 \\ †Department of Chemistry, Chung-Ang University, 84 Heukseok-ro, Dongjak-gu, Seoul 06974, \\ Korea. ¥Korea Research Institute of Standards and Science, 267 Gajeong-ro, Yuseong-gu, \\ Daejeon 34113, Korea \\ *E-mail: sangwoon@cau.ac.kr
}




\section{Dark-Field Scattering Spectra of AuNS-AuNC(face) Dimers from Method A}

The assembly Method A is based on the removal of CTA bilayers on AuNCs using acetonitrile. The CTA bilayers are desorbed by acetonitrile preferentially from the edges or vertices of AuNCs where the SAM structure is less ordered. ${ }^{1}$ Indeed, majority of the AuNS-AuNC dimers are in the on-edge or on-vertex form (Figure 3c). This observation is also consistent with our previous result. ${ }^{2}$ Although very few, however, the on-face isomers are present in the sample prepared by Method A. These isomers seem to form via weak van der Waals interactions between the CTAcovered face of AuNCs and the C8DT-capped AuNSs (Figure S1c). As a result, the interparticle gap distance of these on-face isomers is much longer than the gap distance of the C8DT-linked onface isomers from Method $B(d \approx 4.3 \mathrm{~nm}$ vs. $d \approx 1.3 \mathrm{~nm}){ }^{3,4}$ Accordingly, the longitudinal plasmon coupling mode in the dark-field scattering spectra also appears at a shorter wavelength $(\lambda=710$ $\mathrm{nm}$, Figure S1b), compared to the on-face isomer from Method $B(\lambda=749 \mathrm{~nm}$, Figure $5 b$ ), consistent with the structural characteristics.

(a)

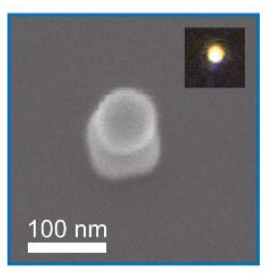

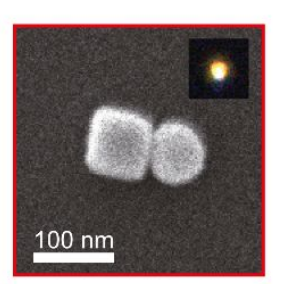

(b)

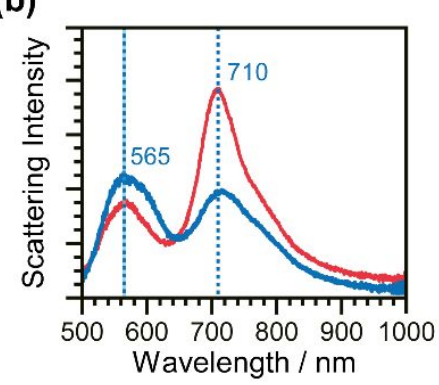

(c)

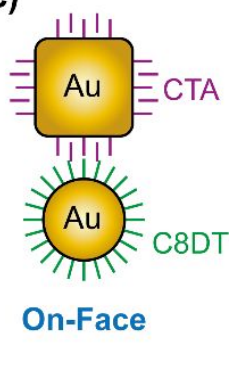

Figure S1. (a) Selected SEM images and (b) correlated dark-field scattering spectra of (c) AuNSAuNC(face) isomers that are prepared by Method A. 


\section{SEM Images and Yield of Dimers from Method A and Method B.}

(a) Method A

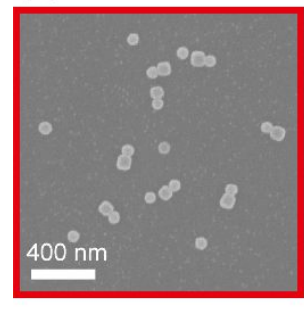

(b) Method B

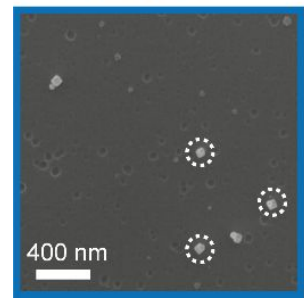

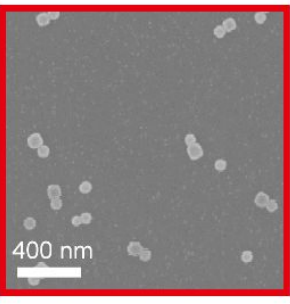

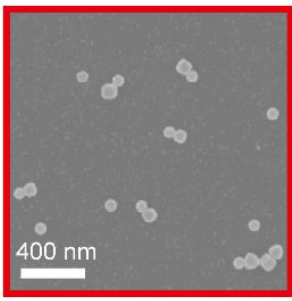

(d)

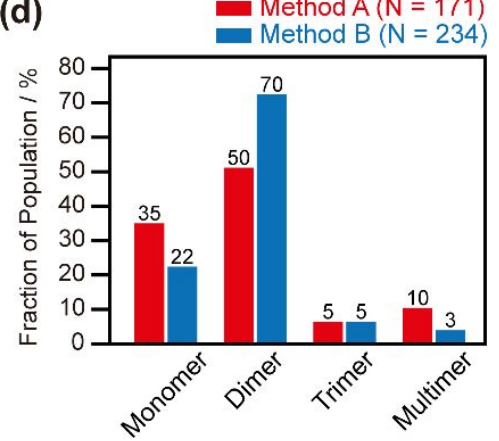

(c)
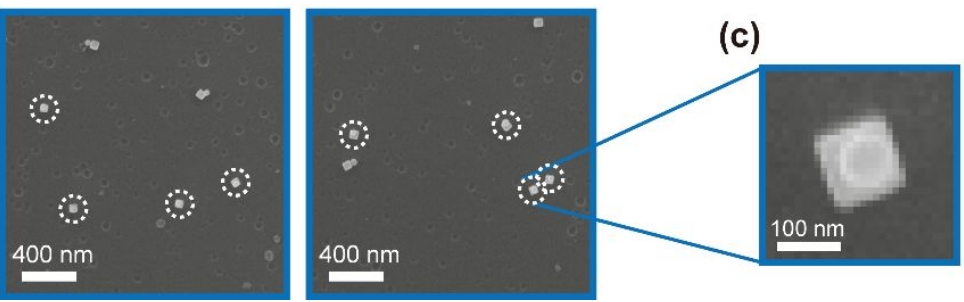

Figure S2. Representative SEM images of the samples prepared by (a) Method A and (b) Method B. (c) Enlarged view of the designated particle in (b). Those nanoparticles that look like AuNC monomers (dotted circles in (b)) are in fact AuNS-AuNC(face) dimers where a AuNS binds on top of the AuNC face, as shown in (c). (d) Percent yields of Method A (red bars) and Method B (blue bars) for monomers, dimers, trimers, and large multimers. 


\section{Distinguishing AuNS-AuNC Isomers}

We tilted the sample stage (up to $25^{\circ}$ ) during the SEM measurements to distinguish AuNSAuNC isomers. Figure S3 below presents a few examples of SEM images acquired by tilting. One should note that it is much easier to make a distinction on the spot. Images become blurrier while they are scanned and saved because of surface charging. We also excluded the dimers in the spectral analysis whose structures were not clearly identifiable.

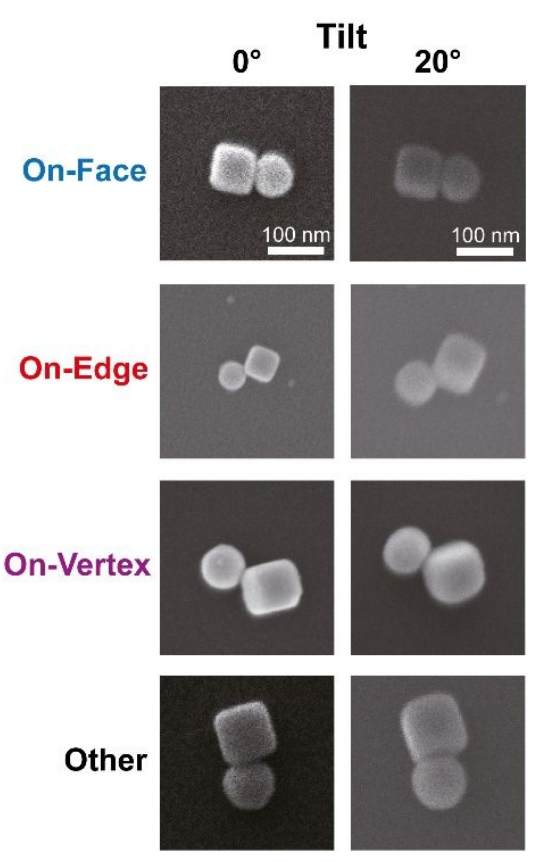

Figure S3. SEM images of on-face, on-edge, and on-vertex AuNS-AuNC dimers acquired before and after tilting the stage by $20^{\circ}$. The bottom image (labeled "Other") corresponds to the dimer structure where a AuNS adsorbs off center of the AuNC face. 


\section{Single-Particle Spectra and the Corresponding Structures}

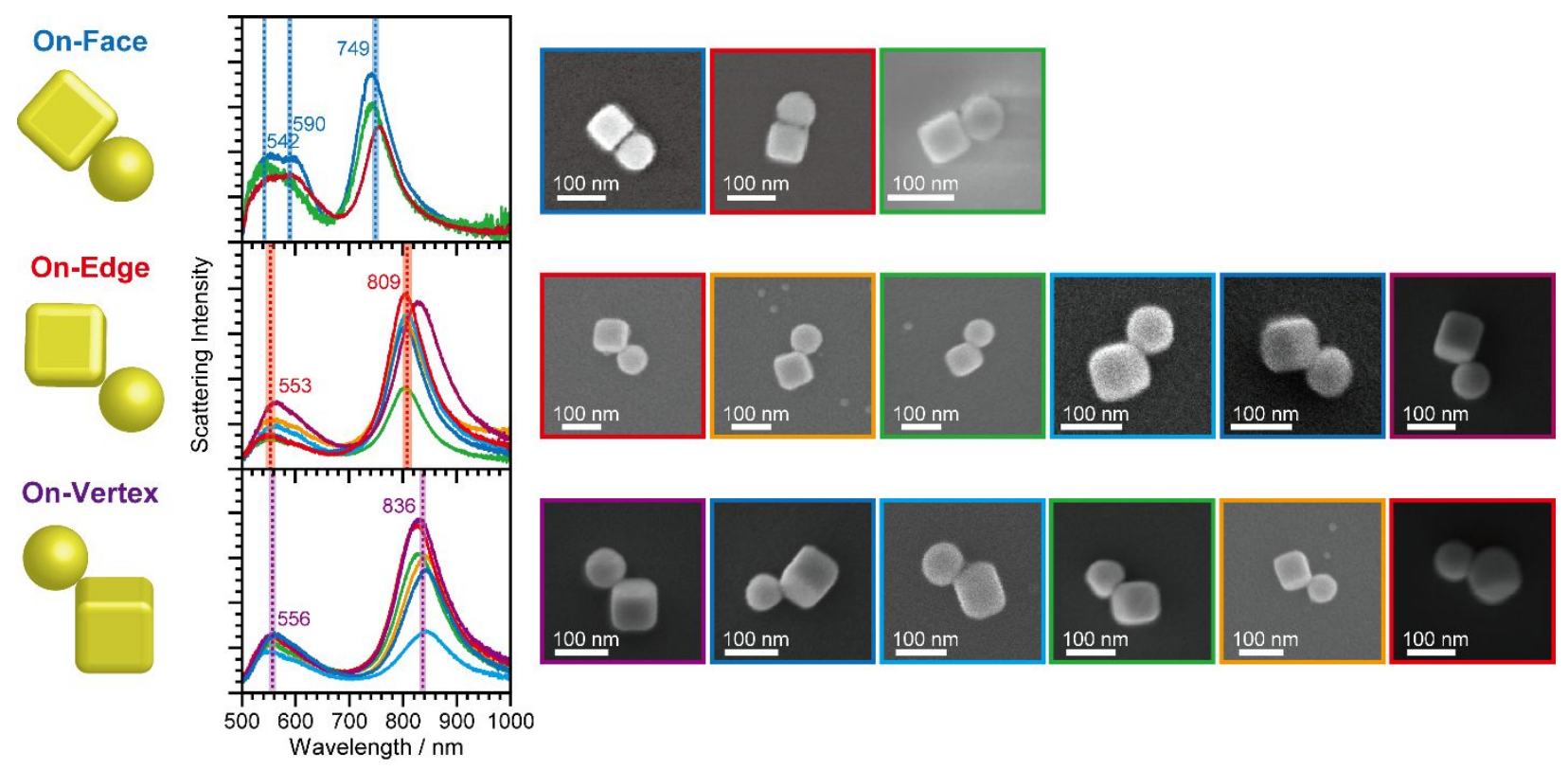

Figure S4. Dark-field single-particle scattering spectra for AuNS-AuNC dimers in three different configurations and their corresponding structures measured by SEM. The spectra and structures are matched by the line colors. 


\section{Simulated Scattering Spectra and Peak Assignments for AuNS-AuNC Isomers}

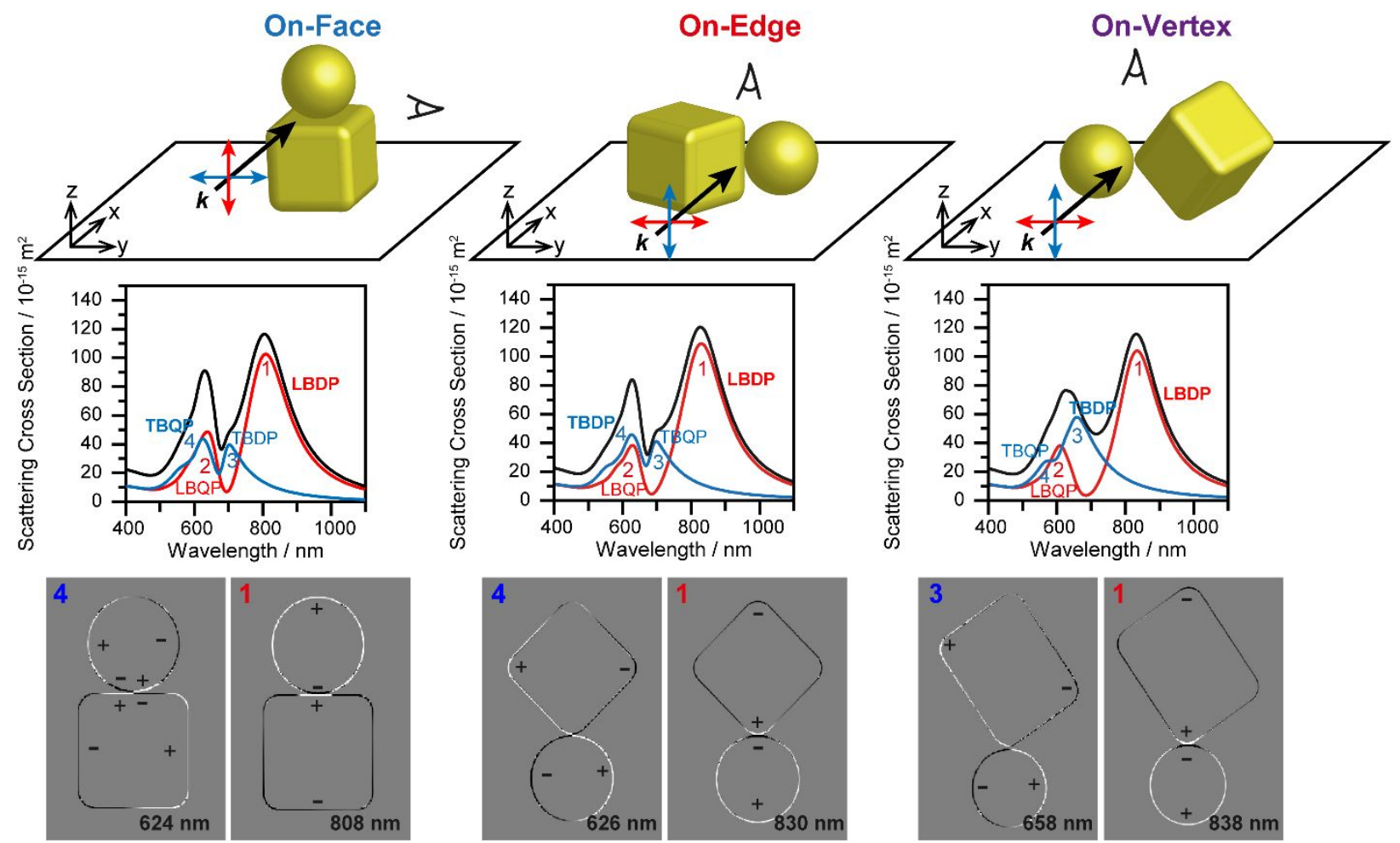

Figure S5. Simulated scattering spectra of AuNS-AuNC isomers excited at the longitudinal (red lines) and transverse (blue lines) polarizations. The plasmon coupling peaks are assigned according to the charge density distributions shown at bottom. $\operatorname{LBD}(\mathrm{Q}) \mathrm{P}$ and $\mathrm{TBD}(\mathrm{Q}) \mathrm{P}$ stand for longitudinal bonding dipole (quadrupole) plasmon and transverse bonding dipole (quadrupole) plasmon, respectively. 


\section{Comparison of Charge Densities and Contact Areas among AuNS-AuNC Isomers}

We calculate the contact area under the assumption that the nanogap $(d=1.3 \mathrm{~nm})$ is maintained up to $20 \%$ of its distance (Figure S6). The increase in the nanogap distance is determined by the high curvature part of the two nanostructures that consist of the nanogap.
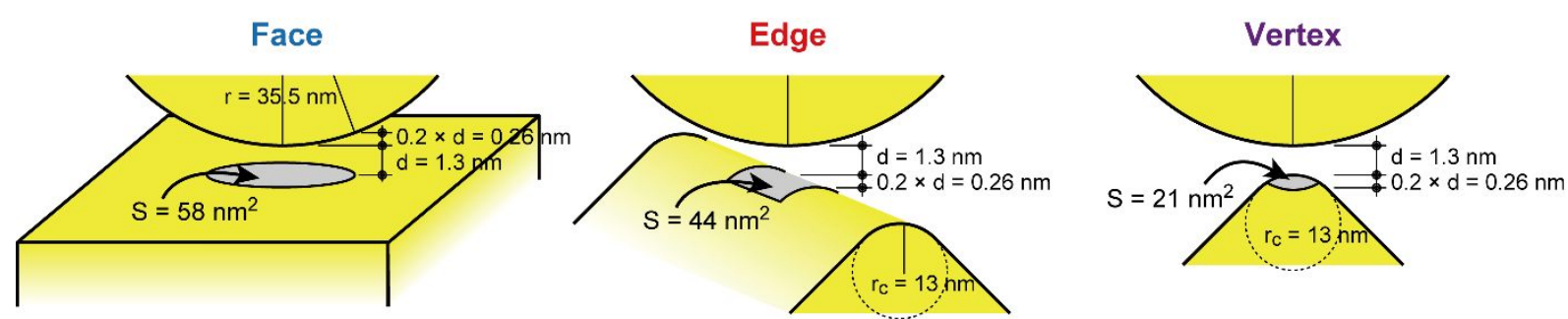

Figure S6. Calculated contact areas (gray color) of the three AuNS-AuNC isomers. We assume that the nanogap properties are maintained up to $20 \%$ of its distance.

We summarize the calculated gap areas and the charge density for each isomer in Table S1 below. As the curvature of the AuNC sharpens, the charge density increases dramatically, especially compared to the decrease in the contact area with the AuNS. Such increases in the Coulomb interaction contribute to lowering the plasmon coupling energy between the vertex of the AuNC and the AuNS.

Table S1. Changes in the charge density and contact area as the curvature of the AuNC sharpens in the AuNS-AuNC nanogaps.

\begin{tabular}{lcccc}
\hline & $\begin{array}{c}\text { Charge Density } \\
{\left[\mathrm{C} \mathrm{m}^{-2}\right]}\end{array}$ & $\begin{array}{c}\text { Relative Charge } \\
\text { Density }\end{array}$ & $\begin{array}{c}\text { Contact Area } \\
{\left[\mathrm{nm}^{2}\right]}\end{array}$ & $\begin{array}{c}\text { Relative Contact } \\
\text { Area }\end{array}$ \\
\hline Face & 0.0023 & 1 & 58 & 1 \\
Edge & 0.22 & 96 & 44 & 0.76 \\
Vertex & 1.06 & 461 & 21 & 0.36 \\
\hline
\end{tabular}




\section{Charge Density Distribution of Icosahedrons}
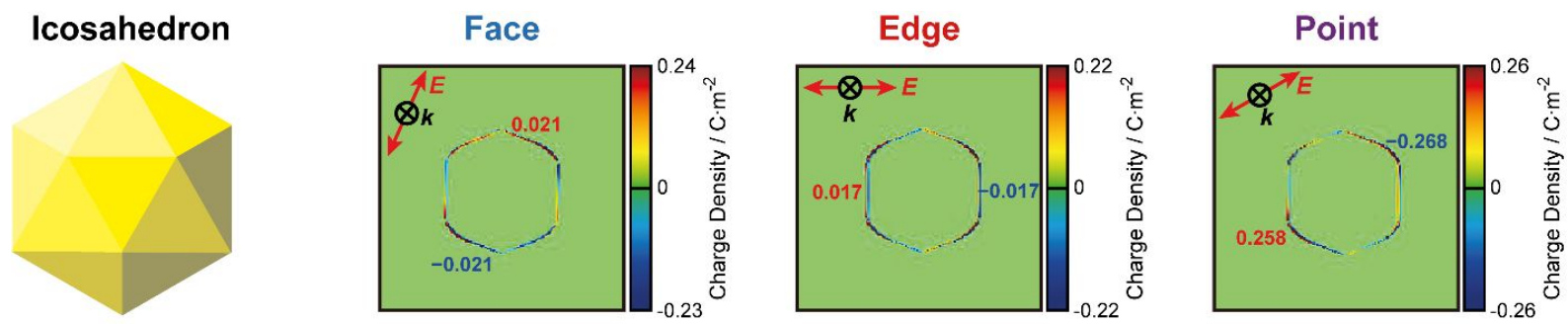

Figure S7. Charge density distribution of an icosahedron excited with the polarization along the face, edge, and point direction. The icosahedron was designed to mimic the faceted AuNPs in the Schlücker group's study (face-face length: $50 \mathrm{~nm}$, curvature radius: $8 \mathrm{~nm}$ ). ${ }^{5}$

Table S2. Relative charge densities of the face, edge, and point of an icosahedron, and calculated contact area between each part of the icosahedron and a AuNS.

\begin{tabular}{lcccc}
\hline & $\begin{array}{c}\text { Charge Density } \\
{\left[\mathrm{C} \mathrm{m}^{-2}\right]}\end{array}$ & $\begin{array}{c}\text { Relative Charge } \\
\text { Density }\end{array}$ & $\begin{array}{c}\text { Contact Area } \\
{\left[\mathrm{nm}^{2}\right]}\end{array}$ & $\begin{array}{c}\text { Relative Contact } \\
\text { Area }\end{array}$ \\
\hline Face & 0.021 & 1 & 41 & 1 \\
Edge & 0.017 & $\mathbf{0 . 8 0}$ & 29 & 0.70 \\
Point & 0.268 & 12.76 & 13 & 0.31 \\
\hline
\end{tabular}




\section{References}

(1) Pramod, P.; Thomas, K. G. Plasmon Coupling in Dimers of Au Nanorods. Adv. Mater. 2008, $20,4300-4305$.

(2) Lee, D.; Yoon, S. Gold Nanocube-Nanosphere Dimers: Preparation, Plasmon Coupling, and Surface-Enhanced Raman Scattering. J. Phys. Chem. C 2015, 119, 7873-7882.

(3) Gómez-Graña, S.; Hubert, F.; Testard, F.; Guerrero-Martínez, A.; Grillo, I.; Liz-Marzán, L. M.; Spalla, O. Surfactant (Bi)Layers on Gold Nanorods. Langmuir 2012, 28, 1453-1459.

(4) Yoon, J. H.; Lim, J.; Yoon, S. Controlled Assembly and Plasmonic Properties of Asymmetric Core-Satellite Nanoassemblies. ACS Nano 2012, 6, 7199-7208.

(5) Yoon, J. H.; Selbach, F.; Schumacher, L.; Jose, J.; Schlücker, S. Surface Plasmon Coupling in Dimers of Gold Nanoparticles: Experiment and Theory for Ideal (Spherical) and Nonideal (Faceted) Building Blocks. ACS Photonics 2019, 6, 642-648. 